\title{
ZU DIESEM HEFT:
}

Zu Beginn des Jahres 1991 beherrschte der Golf-Krieg fast vollständig die Berichterstattung in den Medien. Rüdiger Schlaga erinnert in seinem Beitrag über das Versagen von Politik und Medien im Golf-Krieg daran, wie weitreichend all das, was wir über diesen Krieg erfuhren, bereits durch die Militärzensur gefiltert war. Schlagas Rückblick auf ein bedrückendes Kapitel der Medienwirklichkeit, in der die Medien nicht nur Opfer der Manipulation waren, sondern zu einem beachtlichen Teil auch selbst aktiv die von der Zensur propagierten Informationen, Sprachregelungen und Wertungen der Kriegsereignisse verbreiteten, macht deutlich, daß im Interesse der Sicherung unserer demokratischen Gesellschaft Widerstand gegen jede Art von Zensurmaßnahmen eine sittliche Pflicht des Journalisten ist.

Unter dem Stichwort „Ökojournalismus" lenkt Walter Hömberg in seinem Artikel die Aufmerksamkeit auf einen Bereich journalistischer Arbeit, der angesichts der bestehenden ökologișchen Probleme und der wachsenden Sensibilität der Öffentlichkeit eine zunehmende Bedeutung in den Medien gewinnen wird. Neben offensichtlichen Defiziten und Barrieren des Ökojournalismus, die Hömberg kurz skizziert, geht es ihm aber auch darum zu zeigen, in welcher Weise das Thema "Umwelt" in den Medien sachgerecht und zugleich dem Medium angemessen aufgenommen werden sollte.

Communicatio Socialis veröffentlicht regelmäßig Artikel und Berichte zur Lage der kirchlichen Presse. Im Vordergrund steht dabei zumeist die katholische Presse. Michael Strau $\beta$ versucht mit seinem Artikel, die Konturen eines politischen Mandats für die evangelische Kirchengebietspresse zu zeichnen, indem er den 'Konziliaren Prozeß für Gerechtigkeit, Frieden und Bewahrung der Schöpfung' als Paradigma evangelischer Publizistik bestimmt. Strauß wählt damit einen Problemzugriff, der in seiner ökumenischen Perspektive auch für die katholische Publizistik relevant ist und zu Diskussionen über eine gemeinsame politisch-theologische Standortbestimmung katholischer und evangelischer Publizistik in unserer Gesellschaft anregen kann.

Die Pastoralinstruktion 'Communio et Progressio' bezeichnet Christus als „perfectus communicator" (CeP 11), in der deutschen Übersetzung mit „Meister der Kommunikation" wiedergegeben. Man mag darüber streiten, ob es sich hierbei um eine glückliche Begriffsbildung handelt. Wichtig bleibt der Anspruch, der in dieser Bezeichnung enthalten ist: eine theologische Theorie der Kommunikation christologisch zu fundieren. Diesem Anspruch stellt sich $E d$ mund Arens in seinem Aufsatz "Christus in unsere heutigen Kontexte kommunizieren" und liefert damit einen wichtigen Baustein zu einer bislang noch nicht ausgearbeiteten Theologie der Kommunikation.

Das Thema „Zensur" wird im Berichtsteil dieses Heftes in einem ganz anderen Zusammenhang noch einmal bedeutsam. Ferdinand Oertel legt die Ergebnisse einer Umfrage zur redaktionellen Veranwortung und Zensur in der Kirchenpresse vor. Angesichts des hohen Anspruchs und häufig proklamierten 
Selbstverständnisses der Kirchenpresse machen die von Oertel vorgelegten Umfrageergebnisse doch sehr nachdenklich.

Wie schwierig sich kirchliche Pressearbeit und der kirchliche Umgang mit den Massenmedien in den sich entwickelnden demokratischen Gesellschaften Osteuropas gestalten, zeigen die kurzen Berichte aus Ungarn und Polen. Im Prozeß einer fraglos komplizierten und problemgeladenen gesellschaftlichen Neuorientierung scheint die Kirche zusätzlich noch spezifische Schwierigkeiten zu haben, mit den neu gewonnen Freiheiten wirklich konstruktiv und angstfrei umzugehen.

Auf dem internationalen Kongreß über soziale Kommunikation und Massenmedien 1991 in Wien aus Anlaß des zwanzigjährigen Bestehens von 'Communio et Progressio' (vgl. Communicatio Socialis Heft 3/4, 1991) hatte der Münsteraner Fundamentaltheologe J. B. Metz dafür plädiert, keine Eucharistiefeiern im Fernsehen zu übertragen. Eckhard Bieger hat für Communicatio Socialis daraufhin eine „Replik auf J. B. Metz“ verfaßt, die wir unter der von der Redaktion neu eingeführten Rubrik „ZUR DEBATTE" veröffentlichen.

Helmuth Rolfes 